\title{
Confidence Visual Analogue Scale
}

National Cancer Institute

\section{Source}

National Cancer Institute. Confidence Visual Analogue Scale. NCI Thesaurus. Code C122998.

A scale for the subjective scoring of an individual's confidence that ranges from 1: Not at all confident to 7: Extremely confident. 